\title{
Fetal MRI of hydrometrocolpos with septate vagina and uterus didelphys as well as massive urinary ascites due to cloacal malformation
}

\author{
Yu-Peng Liu • Chih-Ping Chen
}

Received: 22 December 2008 /Revised: 13 January 2009/Accepted: 14 January 2009/Published online: 10 March 2009

(C) Springer-Verlag 2009

A 31-year-old woman at 30 weeks' gestation was referred for evaluation of a fetal pelvic cystic mass found at prenatal US. MRI showed a fluid-filled mass (Fig. 1, arrows) with a midline septum connected to a dilated duplicated uterus (Fig. 1, arrowheads) giving a 'rabbit ears' appearance consistent with hydrometrocolpos with septate vagina and uterus didelphys. There was also massive ascites (Fig. 1, asterisks). The diagnosis was confirmed at autopsy.

Hydrometrocolpos is a rare congenital disorder with cystic dilatation of the vagina and uterus as a result of accumulated secretions from the reproductive tract due to vaginal outflow obstruction, or collected fetal urine due to an obstructed cloacal common channel [1, 2]. Cloacal malformation may be associated with a duplicated genital tract or fetal urinary ascites. The ascites is usually transient. Fetal urine initially drains via the vagina, uterus and fallopian tubes into the peritoneal cavity causing urinary ascites. As the condition progresses, the irritation from the urine and meconium causes obstruction of the fallopian tubes, the development of hydrometrocolpos and disappearance of the ascites [2].

Y.-P. Liu

Mackay Medicine, Nursing, Management College

and Mackay Memorial Hospital,

No 92, Sec. 2, Chung-Shan North Road,

Taipei 104, ROC

e-mail: liupon.liu@msa.hinet.net

\section{C.-P. Chen $(\triangle)$}

Department of Obstetrics and Gynaecology,

Mackay Memorial Hospital,

Taipei, ROC

e-mail: chen@alma.edu
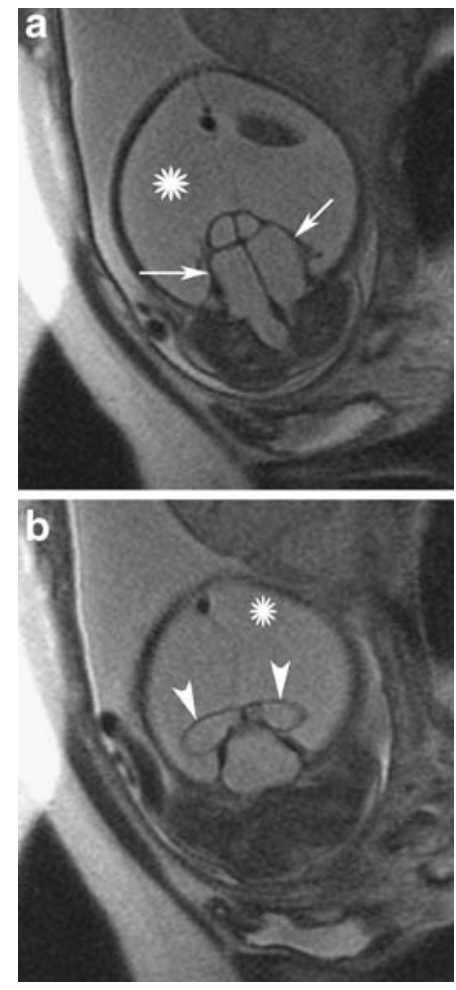

Fig. 1 Coronal T2-W MR images

\section{References}

1. Subramanian S, Sharma R, Gamanagatti S et al (2006) Antenatal MR diagnosis of urinary hydrometrocolpos due to urogenital sinus. Pediatr Radiol 36:1086-1089

2. Gilboa Y, Lipitz S, Zalel Y et al (2007) Prenatal diagnosis of ascites with hydrometrocolpos and uterus didelphys in a monoamniotic twin pregnancy. J Ultrasound Med 26:373-376 